\title{
J-Analyzer: A Software for Computer-Assisted Analysis of Antônio Carlos Jobim's Songs
}

\author{
Carlos Almada $^{1}$, João Penchel ${ }^{1}$, Igor Chagas ${ }^{1}$, Max Kühn $^{1}$, Claudia Usai $^{1}$, Eduardo Cabral ${ }^{1}$, Vinicius Braga ${ }^{1}$, Ana Miccolis ${ }^{1}$ \\ ${ }^{1}$ MusMat Research Group - School of Music - Federal University of Rio de Janeiro \\ Av. Chile, 330/21st floor - 20031-170 - Rio de Janeiro, RJ
}

carlosalmada@musica.ufrj.br

\begin{abstract}
The present paper describes structure and functioning of J-Analyzer, a computational tool for assistedanalysis. It integrates a research project intended to investigate the complete song collection by Brazilian composer Antônio Carlos Jobim, focusing on the aspect of harmonic transformation. The program is used to determine the nature of transformational relations between any chordal pair of chords present in a song, as well as the structure of the chords themselves.
\end{abstract}

\section{Introduction}

This paper is associated with a research project entitled "Systematic studies in popular music", coordinated by Carlos Almada, and developed with the cooperation of seven graduate and undergraduate students, members of the research group MusMat, in the ambit of Federal University of Rio de Janeiro. It describes the structure, functioning, and outputs of a computational program, named $J$ Analyzer, employed as auxiliary tool in the analysis of the complete song collection written by Brazilian composer Antônio Carlos Jobim.

The analysis of Jobim's songs is focused on two specific aspects: binary relations between contiguous chords and the structure of the chords themselves. Some basic questions motivated the elaboration of the project: (a) Which are the most recurrent and, especially, the most peculiar connections between chords used by Jobim? (b) These choices could be due to (at least, partially) voice-leading issues? (c) Which are the chordal qualities employed in this repertoire, considering also cardinality variations? (d) How is their distribution? A in-depth analytical, and original investigation considering these questions (among others) aims at expanding the knowledge in respect to the particular Jobim's harmonic conception, shedding lights also to a neglected field in systematic approaches, namely, structural studies in popular music.

\section{Theoretical framework and background}

The project is theoretically grounded in formulations derived from the Transformational and Neo-Riemannian theories (especially, [1], [2], and [3]), which based an original system (S-J), whose structure was recently described in an international conference on musical theory and analysis [4]. ${ }^{1}$ S-J intends to model Jobim's harmonic practice, through a formal approach addressing both chordal

\footnotetext{
${ }^{1}$ For some studies related to a previous version of the current system, see [5], [6], and [7].
}

structures and binary relations. Basically, the system operates through the notion of classes of chordal quality, or more simply, chordal classes. Basically the classes are archetypical, abstract structures that represent tonal chords through correspondences with their internal structures, which define their respective qualities. We consider ten classes of chordal qualities: major with major seventh (labelled as Z), ${ }^{2}$ dominant seventh (Y), French-sixth ${ }^{3}(\mathrm{X})$, augmented-fifth dominant (W), minor with seventh (z), half-diminished $(y)$, diminished seventh $(x)$, minor with major seventh (w), as well as major (V) and minor triads (v). ${ }^{4}$ These basic classes in turn contain subsets formed by variants (for example, the dominant qualities "with flat nine", "with thirteenth", and "substitute fourth" are three members of the class Y).

The classes are derived through application of a group of special functions, algebraically, and geometrically ${ }^{5}$ formalized. The subclasses are derived from the classes through application of transformational operations. ${ }^{6}$

\section{Pre-analytical considerations}

The analysis of each song is preceded by four stages:

- Transcription of the vocal and piano $\operatorname{score}^{7}$ as a file in MusicXml format;

- Revision of the transcription, searching for errors and incongruities of any type, concerning the harmonic aspect;

- Rewriting of the chords (maintaining their original metric positions) as normal-form structures, i.e., in the most compact disposition (inside one octave), and always in root position;

- Storing of the normal-form harmonic sequence as a midi file, for further analysis in the program JAnalyzer.

The scheme of Figure 1 summarizes the four stages of the pre-analytical process.

\footnotetext{
${ }^{2}$ We adopt the final letters of the alphabet, in reverse order, for labelling the classes in order to not confuse with the initial letters (A-G), employed for naming chordal roots.

${ }^{3}$ Or dominant chord with flat fifth

${ }^{4}$ Both classes are left at the end of the list because triads are relatively uncommon in Jobim's harmony.

${ }^{5}$ The geometric projections of the chordal classes is made on an original planar scheme, named Tetranetz.

${ }^{6} \mathrm{~A}$ detailed explanation about theoretical aspects of S-J is presented in [8].

${ }^{7}$ We adopt as reference for the transcription process the five volumes of the Cancioneiro Jobim [9].
} 


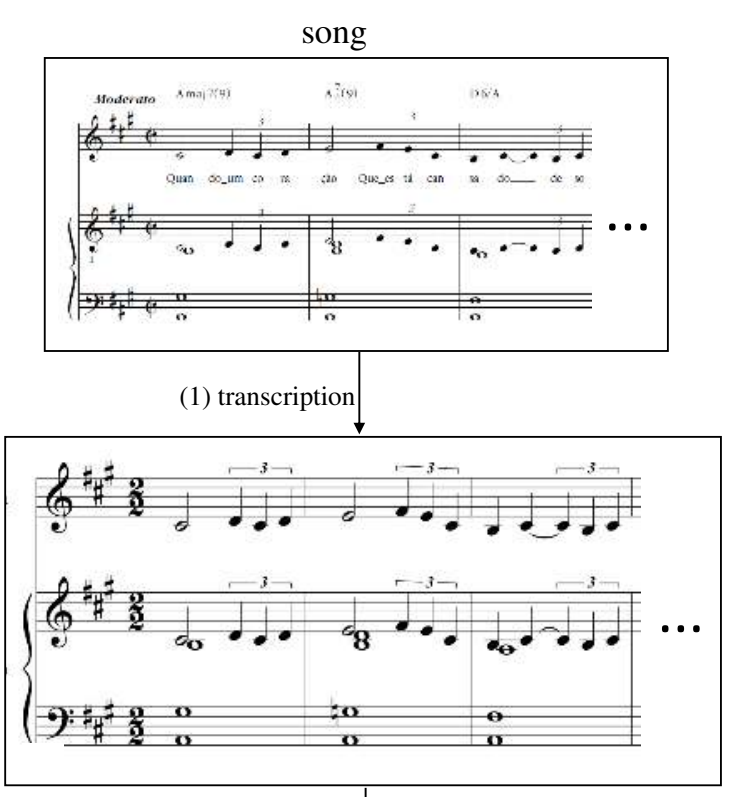

(2) revision

(3) normal-form harmony

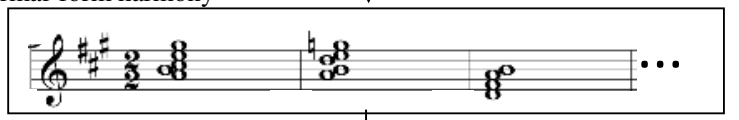

(4) storing
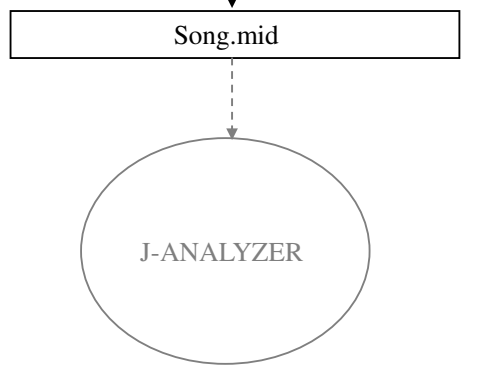

Figure 1: Representation of the four preanalytical stages considering one of Jobim's song.

\section{Functioning and structure of J-Analyzer}

J-Analyzer was implemented in Matlab language, encompassing a set of complementary and/or sequential embedded functions and scripts. Since binary relations is one of the central aspects concerned in the analytical process, J-Analyzer works with a two-chord window per turn (i.e., chords 1-2, 2-3, 3-4, ..., (n-1)-n). For each pair of chords selected J-Analyzer returns their pitch-class content, chordal cardinalities (i.e., the number of elements), the set of common pitch classes, roots (expressed also as pitch classes), root distance (as number of semitones, between -5 and 6), class/subclass formal labels, and finally a binary-transformational operational designation, which expresses precisely, and concisely not only the relation between the two chords, but also their internal structure. Each chordal class or subclass registered in the system is named according to a simple convention: instances of the basic ten classes, above described, are labelled with the corresponding letter followed by a "zero" (for example, Z0, representing any major chord with major seventh, does not mattering which specific root is in question); variants of the classes (i.e., subclasses) are identified by a sequential number different from zero, associated with the corresponding letter (e.g., Z1, for the subclass that represents all major chords with major seventh and major ninth). Figure 2 presents a basic overview of the analytical output, taking as example the chordal progression DMaj7-Gm.

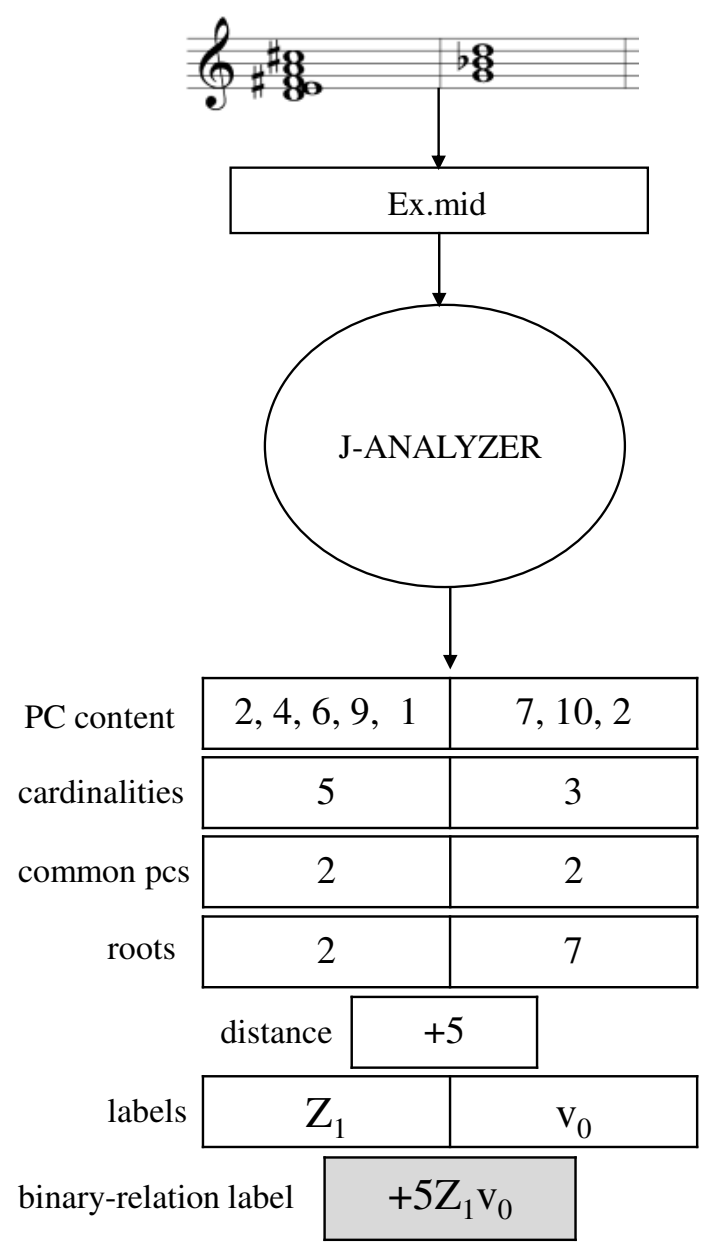

Figure 2: Example of output of J-Analyzer.

The functioning of J-Analyzer is essentially simple and can be described as a sequence of some steps, following the opening of the midi file corresponding to the song to be analyzed:

- Conversion of the midi information into a numeric matrix (or $n m a t$ ) $i \times 7,{ }^{8}$ where $i$ corresponds to the number of events present in the song (Figure 3 shows a short excerpt of the nmat corresponding to the normal-form harmony of Garota de Ipanema (Tom Jobim and Vinícius de Moraes), as illustration). The columns refer to, respec-

\footnotetext{
${ }^{8} \mathrm{~A}$ nmat is a numeric matrix produced by the function midi2nmat (which integrates a MIDI toolbox created by the researchers Tuomas Eerola and Petri Toiviainen, from Jyväskylä University, Finland).
} 
tively, (1) beat positions of the onsets (considering 1 as a quarter note and 0 as the starting point), (2) durations (in beat multiples or sub-multiples), (3) track numbers, (4) pitches (in midi-pitch numbers), (5) velocity, (6) time points (measured in seconds), and (7) durations (also in seconds);

\begin{tabular}{|c|c|c|c|c|c|}
\hline 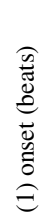 & 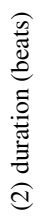 & $\stackrel{\frac{y}{\tilde{E}}}{\stackrel{\Xi}{\Xi}}$ & 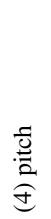 & $\begin{array}{l}\frac{2}{0} \\
\frac{0}{0} \\
\stackrel{2}{D}\end{array}$ & $\begin{array}{l}\text { ô } \\
\text { : } \\
0 \\
0 \\
0 \\
0 \\
0 \\
0 \\
0 \\
0 \\
0 \\
0\end{array}$ \\
\hline 0 & 4 & 3 & 65 & 69 & 0 \\
\hline 0 & 4 & 3 & 69 & 69 & 0 \\
\hline 0 & 4 & 3 & 72 & 69 & 0 \\
\hline 0 & 4 & 3 & 76 & 69 & 0 \\
\hline 8 & 4 & 3 & 67 & 70 & 4 \\
\hline 8 & 4 & 3 & 71 & 70 & 4 \\
\hline 8 & 4 & 3 & 74 & 70 & 4 \\
\hline 8 & 4 & 3 & 76 & 70 & 4 \\
\hline 8 & 4 & 3 & 77 & 70 & 4 \\
\hline 12 & 4 & 3 & 60 & 70 & 6 \\
\hline 12 & 4 & 3 & 61 & 70 & 6 \\
\hline 12 & 4 & 3 & 65 & 70 & 6 \\
\hline 12 & 4 & 3 & 67 & 70 & 6 \\
\hline 12 & 4 & 3 & 70 & 70 & 6 \\
\hline
\end{tabular}

Figure 3: First fifteen lines of Garota de Ipanema (Jobim and Moraes) harmonic nmat.

- Because only pitch and onset information are relevant in the analysis, both columns 1 and 4 are extracted from the nmat and merged in a new matrix of dimensions ix 2 . Pitches are then converted into pitch classes (through simple modulo 12 operation). Since the exact position of the chords does not matter for the analysis, but their relative positions (first, second, etc.), a new matrix is created, depicting vertically, and sequentially the chords as pitch classes in normal-form disposition (Figure 4);

- The program selects then the two first chords for analysis. Roots, cardinalities and pitch-class contents are automatically obtained. After stored (for further statistical purposes), each normal-form ordered set is converted into a prime form, by subtracting in mod12 the pitch-class root from its content (or else, for convention, any prime form has root transposed to pitch class 0 ). The intention behind this normal-prime convertion is to obtain a still more abstract representation of each chord, which fits the goals of the analytical investigation. The intervallic configuration of the prime form is then codified as an unique integer by the function "pcset2Goedel", which implements an adaptation of the algorithm known as Gödel Numbering. Initially, this algorithm arbitrates for each symbol of a string formed by $n$ typografic signs (originally, a logical proposi-

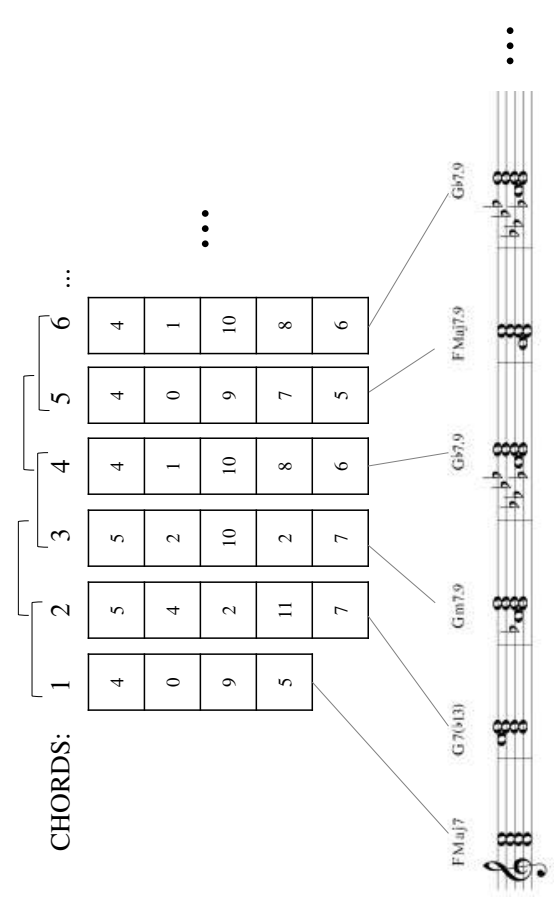

Figure 4: First six chords of Garota de Ipanema in pitch-class notation and normal form. Brackets indicate pairs of chords that will be considered for the subsequent analysis.

tion of the Theory of Numbers) a numeric code. The codified, ordered numbers (that substitute for the string's symbols) are then considered as exponents raising a sequence of the $n$ first prime numbers. The product of the raised primes results in a (normally very big) integer, an univocal index ${ }^{9}$ that, ultimately, represents the original proposition (its factoring returns exactly the same sequence of code numbers, which can then be translated to the initial symbols). ${ }^{10}$ In our system S$\mathrm{J}$, for a Jobinian prime-form chord of cardinality $n$, instead of using directly their $\mathrm{n}$ pitch classes, the algorithm extracts the sequence of their intervals, forming a vector of $n-1$ elements, treated as the exponents that raise the sequence of $n-1$ first primes (because the intervals are expressed directly as numbers, the phase of translation of symbols is here bypassed). There are two good reasons for use intervals, rather than pitch classes as exponents: (1) conciseness, due to the use of one less element, and especially lower-value ex-

\footnotetext{
${ }^{9}$ This argument is based on the Fundamental Theorem of Arithmetic, which states that any integer greater than 1 is a prime number or it is the result of the product of prime numbers.

${ }^{10}$ This extremely elegant and clever algorithm was elaborated by Austrian mathematician and logician Kurt Gödel (1906-1978), associated with which is now known as his Incompleness Theorems. For more detailed information about Gödel, his algorithm, and the revolutionary aspect of his contributions to the mathematic logic, see [10]. For another musical application of Gödel Numbering, see [11].
} 
ponents (both contributing for Gödel numbers of lower magnitude), and (2) the fact that the intervallic vector represents the very unique structure of the chord which with is associated, a merit in itself. The integer that results from the raisedprime product is considered the Gödel number (G) associated with the prime-form chord in question (a strictly one-to-one relation). In other words, there are in the system as many unique Gs as there are classes and subclasses of qualities. The two Gs are then compared with a previouslyprepared lexicon of chordal class and subclasses, and their definitive formal labels are obtained. It is noteworthy mention that another incorporated function (pc2label) translates normal forms into chordal labels in informal notation (in the case of the example, "FMaj7.9" and "G7.13"), which are then displayed in an output list and stored for further statistical analysis. Figure 5 summarizes the sequence of events in this stage;

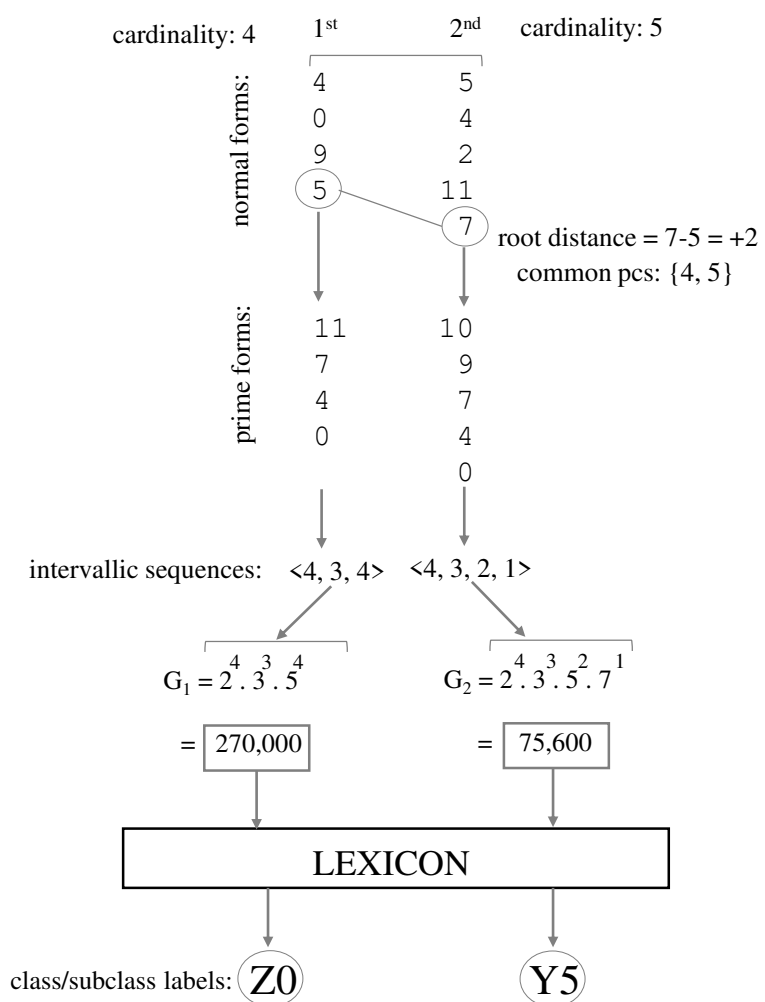

Figure 5: Outputs from the analysis of the first chordal binary relation of Garota de Ipanema: normal and prime forms, common pitch classes, roots, root distance, intervallic sequences, Gödel numbers, and class/subclass formal labels.

- Finally, the operation label related to the transformational connection of the two chords (or, in formal terms, the binary-transformational operation) is produced, being formatted as a string, by assembling root distance, and the two quality formal labels (preserving the same order of apparition of the chords). In the case exemplified, the operation is identified as the string +2Z0Y5. Similarly what was made for the intervallic sequences, the operation label is also converted in a Gödel number, which is then stored. However, this case has two particularities that deserve to be mentioned: (a) since the string has always the same cardinality of six fixed positions (direction-interval-letter-numberletter-number), the sequence of prime bases is consequently also fixed: $<2,3,5,7,11,13\rangle$; and (b) beside numbers, other symbols are also used in the string, which implies that a previous translation stage is necessary. Concerning this, codes were assigned to the non-numeric symbols used, as shown in Table 1. These symbols (that occupy the odd positions of the string) were subdivided according to the categories which with are associated: intervallic direction ( 0 or 1 , respectively, for ascending or descending), seventh chords with major quality ( $\mathrm{Z}$ to $\mathrm{W}, 0$ to 3$),{ }^{11}$ seventh chords with minor quality ( $\mathrm{z}$ to w, 4 to 7), and triads ( $\mathrm{V}$ and v, 8 and 9). Even positions are filled by numbers (i.e., interval sizes and order numbers of classes/subclasses), and therefore represent themselves in the translated sequence. Thus, the string corresponding to the first couple of chords of the example is numerically encoded as follows:

$+2 \mathrm{Z} 0 \mathrm{Y} 5 \equiv<0,2,0,0,1,5\rangle$, which become exponents for the six prime bases, from whose product is obtained the corresponding Gödel number $\mathrm{G}_{1-2}$ (i.e., referred to the binary relation between chords 1 and 2):

$G_{1-2}=2^{0} \cdot 3^{2} \cdot 5^{0} \cdot 7^{0} \cdot 11^{1} \cdot 13^{5}=36,758,007$

In spite of its magnitude, $G_{1-2}$ is stored by $\mathrm{J}$-Analyzer (together with the remaining indices that represent the following binary relations). When necessary the information related to these huge numbers are easily retrieved through prime factoring and subsequent translation.

- After concluding the analysis of the first binary relation, another pair of chords (numbers 2 and 3, in this case) is then selected and the whole analytical process is repeated by the program, and so on, until the end of the harmonic progression (chords $\mathrm{n}-1$ and $\mathrm{n}$ ). The complete set of data is stored in matrices. A list summarizing the main information for the examination by the user/analyst is then produced as a visual output of $\mathrm{J}$-Analysis (Figure 6 adapts a brief portion of the list referred to the analysis of Garota de Ipanema). ${ }^{12}$

\footnotetext{
${ }^{11}$ Because symbols for direction are exclusively settled at the first position of the string, codes 0 and 1 can be also attributed to the quality symbols, that form the third and fifth positions.

${ }^{12}$ Because of their great magnitudes, the Gödel numbers of prime forms and operations are not displayed in the list.
} 
Table 1: Encoding of symbols used in the binarytransformational operations.

\begin{tabular}{ccc}
\hline \hline symbol & meaning & code \\
\hline+ & ascending interval & 0 \\
- & descending interval & 1 \\
\hline $\mathrm{Z}$ & major w/ major seventh & 0 \\
$\mathrm{Y}$ & dominant seventh & 1 \\
$\mathrm{X}$ & French-sixth & 2 \\
$\mathrm{~W}$ & augmented dominant & 3 \\
\hline $\mathrm{Z}$ & minor w/ seventh & 4 \\
$\mathrm{y}$ & half-diminished & 5 \\
$\mathrm{X}$ & diminished seventh & 6 \\
$\mathrm{w}$ & minor w/ major seventh & 7 \\
\hline $\mathrm{V}$ & major triad & 8 \\
$\mathrm{v}$ & minor triad & 9 \\
\hline
\end{tabular}

\section{Concluding remarks}

This paper presented basic structure and functioning of the program J-Analyzer, a computer-assisted tool used in an in-depth, original analysis of Jobim's songs, addressing transformational harmonic aspects. With 110 songs already analyzed (out a total of 146), the first phase of the research is next to its conclusion. J-Analyzer can be considered as a powerful tool for the tasks to which was designed, namely, conversion of midi file into individual chordal information (essentially, structure, cardinality, and class/subclass type), and the transformational relations that connect each pair of them. More specifically, the strategy of adaptation Gödel-numbering function to the encoding of essential information for the analysis proved highly effective, since the compaction of numeric sequences and symbolic strings into univocal integers contributes to economy in the storing of data as well as facilitates retrieval processes.

As next stages of the present research, the multitude of data produced in the analyzes will be combined, crossed and interpreted, through the help of another software, JStatistics, considering not only the complete repertoire of songs, but also their subdivision into the so-considered five creative periods of Jobim (1947-58; 59-65; 66-70; 71-82; and 83-94), in order to evidence tendencies, particularities, and intersections of his harmonic preferences.

\section{References}

[1] LEWIN, D. (1987). Generalized Musical Intervals and Transformations. New Haven: Yale University Press.

[2] KOPP, D. (2002). Chromatic Transformations in Nineteenth-Century Music. New York: Cambridge University Press.

[3] RINGS, S. (2011). Tonality and Transformation. Oxford: Oxford University Press.

[4] ALMADA, C. etal (2019). An Analytical Methodology for Harmonic Analysis of Antônio Carlos Jobim's Songs. International Symposium on the Analysis and Theory of Music, 1., Proceedings. Porto.

[5] ALMADA, C. (2017a). Uma proposta teórica visando à aplicação de princípios neorriemanianos em música popular. Congresso da Associação Nacional de Teoria e

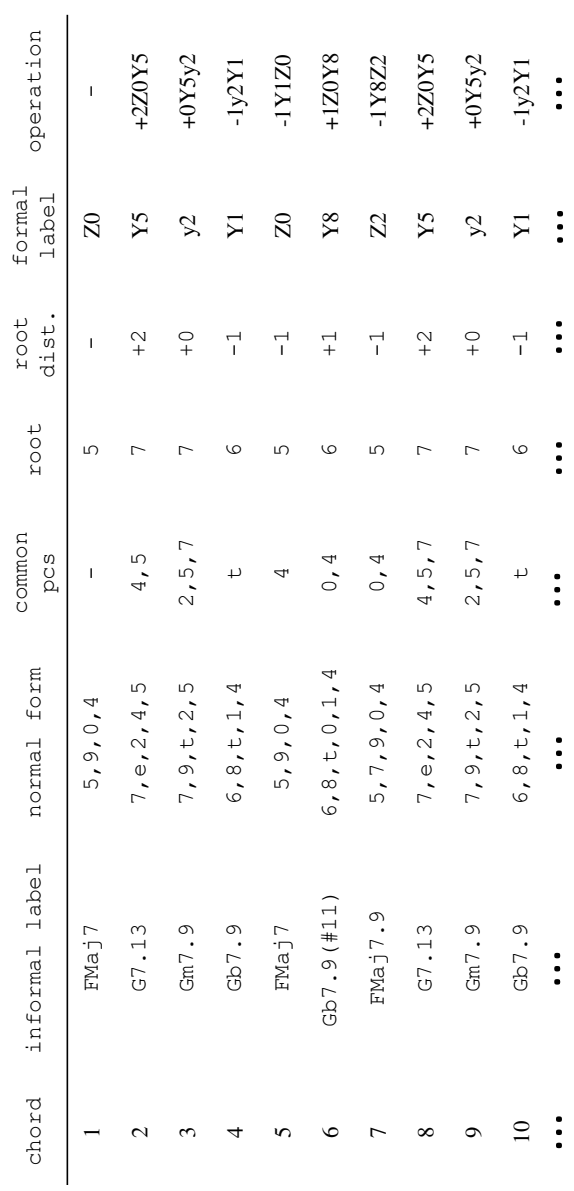

Figure 6: Partial view of the data-list of Garota de Ipanema, considering numbers of the chords, informal labels of the chords, normal forms, sets of common pitch classes, roots, root distances, quality formal labels, and binary-operation labels.

Análise Musical, 4., Proceedings. Florianópolis: UDESC, 20-30.

[6] ALMADA, C. (2017b). Representação geométrica de conduções parcimoniosas de vozes emprogressões harmônicas em música popular. Encontro Nacional da ANPPOM, 27., Proceedings. Campinas: UNICAMP.

[7] KUHN, M. etal (2017). Relações neorriemanianas de acordes de sétima na primeira fase composicional de Antônio Carlos Jobim. Encontro Internacional de Teoria e Análise Musical, 4., Proceedings. São Paulo: USP, 147-183.

[8] ALMADA, C. etal (2018). Uma nova abordagem referente à ocorrência de transformações harmônicas em canções de Antônio Carlos Jobim. Congresso Brasileiro de Música e Matemática, 3., Proceedings. Rio de Janeiro: UFRJ (in press).

[9] JOBIM, A. (2006). Cancioneiro Jobim: Obras escolhidas. Rio de Janeiro: Instituto Antônio Carlos Jobim (Score).

[10] NAGEL, E. and NEWMAN, J. (2001). Gödel's Proof. New York: New York University Press.

[11] ALMADA, C. (2017b). Gödel-Vector and GödelAddress as Tools for Genealogical Determination of Genetically-Produced Musical Variants. In: The MusicalMathematical Mind: Patterns and Transformations. Cham (Switzerland): Springer, 9-16. 